S. Mori

Nagoya Math. J.

Vol. 96 (1984), 127-132

\title{
ON DEGREES AND GENERA OF CURVES ON SMOOTH QUARTIC SURFACES IN $P^{3}$
}

\author{
SHIGEFUMI MORI
}

Our result is motivated by the results [GP] of Gruson and Peskin on characterization of the pair of degree $d$ and genus $g$ of a non-singular curve in $\boldsymbol{P}^{3}$. In the last step, they construct the required curve $C$ on a singular quartic surface when $g \leqq(d-1)^{2} / 8$. Here we consider curves on smooth quartic surfaces.

The author expresses his hearty thanks to Professor Hartshorne who pointed out a mistake in the earlier version of this paper, and to Professor litaka who kindly allowed the author to use his word processor to write up this paper.

THEOREM 1. Let $k$ be an algebraically closed field of characteristic 0 and $d>0$ and $g \geqq 0$ be integers. Then there is a non-singular curve $C$ of degree $d$ and genus $g$ on a non-singular quartic surface $X$ in $P^{3}$ if and only if (1) $g=d^{2} / 8+1$, or (2) $g<d^{2} / 8$ and $(d, g) \neq(5,3)$.

Remark 2. Under the notation of Theorem $1, g=d^{2} / 8+1$ if and only if $C$ is a complete intersection of $X$ and a hypersurface of degree $d / 4$, which will be proved in the proof below.

Proof of the only-if-part $(\Rightarrow)$ of Theorem 1. Let $H=\mathcal{O}_{X}(1)$. Since $(H \cdot H)>0$, one has

$$
(C \cdot H)^{2}-(H \cdot H) \cdot(C \cdot C)=d^{2}-8(g-1) \geqq 0,
$$

by Hodge index theorem, because $X$ is a $K 3$ surface and $K_{C}=\mathcal{O}_{c}(C)$. One has $d^{2} \equiv 0,1,4,1(\bmod 8)$ according as $d \equiv 0,1,2,3(\bmod 4)$. If $d^{2}-8(g-1)=0$ then the classes of $\mathcal{O}_{X}(C)$ and $\mathcal{O}_{X}(H)$ are proportional. Since $X$ is a $K 3$ surface and $(H \cdot H)=4$, Pic $X$ is torsion-free and $H$ is not divisible, whence $\mathcal{O}_{X}(C)$ is a multiple of $\mathcal{O}_{X}(H)$, which implies that $C$ is a complete intersection of $X$ and a hypersurface of degree $d / 4$. It

Received November 17, 1983. 
remains to show that $d^{2}-8(g-1)>8$ when $d^{2}-8(g-1)>0$, and we will treat three cases $d^{2}-8(g-1)=8,1,4$.

Case $(1) d^{2}-8(g-1)=8: \quad$ Let $d=4 d^{\prime}\left(d^{\prime} \geqq 1, d^{\prime} \in Z\right)$, then $2(g-1)$ $=2\left(2 d^{\prime 2}-1\right)$. Let $E=d^{\prime} H-C$, then $(E \cdot H)=0$ and $\left(E^{2}\right)=-2$. Since $X$ is a $K 3$ surface, one has

$$
h^{0}(E)+h^{0}(-E) \geqq \chi(\mathcal{O}(E))=2+\left(E^{2}\right) / 2=1 .
$$

Thus $E$ or $-E$ gives a curve $E^{\prime}$ such that $\left(E^{\prime} \cdot H\right)=0$, which contradicts the very ampleness of $H$.

Case (2) $d^{2}-8(g-1)=1$ : Let $d=2 d^{\prime}-1\left(d^{\prime} \geqq 1, d \in Z\right)$, then $2(g-1)=\left(d^{\prime 2}-d^{\prime}\right)$. Let $E=d^{\prime} H-2 C$, then $(E \cdot H)=2$, and $\left(E^{2}\right)=0$.

Thus as in Case (1), $h^{0}(E)+h^{0}(-E) \geqq 2$. Since $(E \cdot H)=2$ for ample $H$, one has $h^{\circ}(-E)=0$ and $h^{0}(E) \geqq 2$. Thus $|E|$ has an effective member $E_{0}$. If $E^{\prime}=\left(E_{0}\right)_{\text {red }}$ is irreducible, then $\left(E^{\prime 2}\right)=0$ and $\left(E^{\prime} \cdot H\right)=1,2$ for very ample $H$. Thus $E^{\prime} \cong P^{1}$ and $\left(E^{\prime 2}\right)=-2$, which contradicts $\left(E^{\prime 2}\right)=0$. Hence $\left(E_{0}\right)_{\text {red }}$ is reducible and by $\left(E_{0} \cdot H\right)=2$ for very ample $H$, one has $E_{0}=E_{1}+E_{2}$, where $E_{1}, E_{2} \cong P^{1}, \sharp\left(E_{1} \cap E_{2}\right) \leqq 1$, and the intersection of $E_{1}$ and $E_{2}$ is transverse. Then $\left(E_{0}^{2}\right)=-4+2\left(E_{1} \cdot E_{2}\right) \leqq-2$, which contradicts $\left(E_{0}^{2}\right)=0$.

Case (3) $d^{2}-8(g-1)=4:$ Let $d=4 d^{\prime}-2\left(d^{\prime} \geqq 1, d^{\prime} \in Z\right)$, then $2(g-1)=4\left(d^{\prime 2}-d^{\prime}\right)$. If $E=d^{\prime} H-C$, then $(E \cdot H)=2$ and $\left(E^{2}\right)=0$. Thus one gets a contradiction as in Case (2). If $d=5$ and $g=3$, then $d>2 g-2$. Thus $h^{0}\left(\mathcal{O}_{C}(1)\right)=3$, which implies that $C$ is a plane curve, but this contradicts the genus formula for plane curves. Thus " $\Rightarrow$ " is proved.

Proposition 3. Let $d$ and $g$ be integers such that $0 \leqq g \leqq d-3$. If char $k \neq 2$, then there exist a non-singular Kummer surface $X_{0}$ and effective divisors $H_{0}, C_{0}$ on $X_{0}$ such that

(1) $\left(H_{0}^{2}\right)=4,\left(H_{0} \cdot C_{0}\right)=d,\left(C_{0}^{2}\right)=2 g-2$,

(2) $H_{0}$ is numerically effective,

(3) $C_{0}$ is numerically effective if $g \geqq 2$,

(4) $Z H_{0}+Z C_{0}$ is a direct summand of Pic $X_{0}$.

Proof. Let $k=d-g-3 \geqq 0$. Let $Y_{1}$ and $Y_{2}$ be elliptic curves with an isogeny $f: Y_{1} \rightarrow Y_{2}$ of degree $2 k+1$. Let $P, Q \in Y_{1}$ be non-zero points such that $2 P=0, f(2 Q) \neq 0$. Let $X_{0}$ be the non-singular Kummer surface 


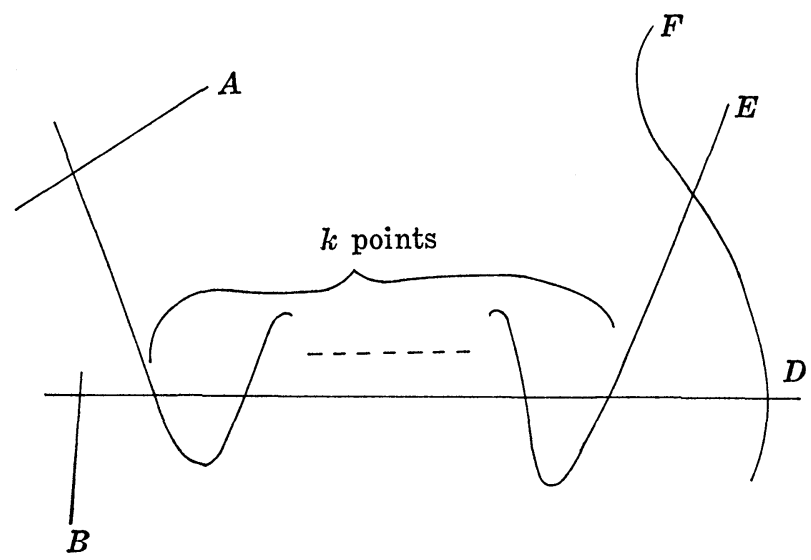

associated to $Y_{1} \times Y_{2}$. Then $Y_{1} \times 0, Q \times Y_{2}$, the graph of $f, P \times f(P)$, and $P \times 0$ give irreducible curves $D, F, E, A$, and $B$ in $X_{0}$ such that $D \cong E \cong$ $A \cong B \cong P^{1}$, and $F$ is an elliptic curve, with the configuration as in the picture with all the intersections transverse (cf. [MM] or [SI]). Let $H_{0}$ $=D+3 F$, and $C_{0}=E+g F$. Then (1) is clear; (2) follows from $\left(H_{0} \cdot D\right)$ $=1$ and $\left(H_{0} \cdot F\right)=1$; (3) follows from $\left(C_{0} \cdot E\right)=g-2$ and $\left(C_{0} \cdot F\right)=1$; and (4) follows from $\left(H_{0} \cdot B\right)=1,\left(H_{0} \cdot A\right)=0,\left(C_{0} \cdot B\right)=0$, and $\left(C_{0} \cdot A\right)=1$. q.e.d.

Remark 4. Let $k$ be the field of complex numbers. Then, in the local versal deformations space Def of $X_{0}$, the locus where $H_{0}$ and $C_{0}$ lift as line bundles is an 18-dimensional smooth subvariety Pol, and there is a dense subset $\mathrm{Pol}^{\prime}$ of $\mathrm{Pol}$ such that if $q \in \mathrm{Pol}^{\prime}$, then the surface $X$ and line bundles $H$ and $C$ on $X$ lying over $q$ satisfy the conditions:

(1) $\left(H^{2}\right)=4,(H \cdot C)=d,\left(C^{2}\right)=2 g-2$,

(2) $H$ is numerically effective,

(3) $C$ is numerically effective if $g \geqq 2$, and

(4) $\operatorname{Pic} X=Z H+Z C$.

Indeed (1) is clear, whence $X$ is algebraic by [K, Theorem 8], and (4) follows from [K, Theorem 14]. As for (2) and (3), $2 H_{0}$ and $2 C_{0}$ (if $g \geqq 2$ ) are base point free by (1) of Theorem 5. The obstructions for lifting sections of $\mathcal{O}\left(2 H_{0}\right)$ and $\mathcal{O}\left(2 C_{0}\right)$ (if $g \geqq 2$ ) to Pol lie in $H^{1}\left(\mathcal{O}\left(2 H_{0}\right)\right.$ ) and $H^{1}\left(\mathcal{O}\left(2 C_{0}\right)\right)$ which are both 0 by Ramanujam's vanishing theorem.

We now quote results by Saint-Donat:

Theorem 5 (Saint-Donat [SD] or cf. [MM]). Let $X$ be a K3 surface defined over an algebraically closed field of characteristic $\neq 2$. Let $H$ be 
a numerically effective divisor on $X$. Then one has

(1) $H$ is not base point free if and only if there exist irreducible curves $E, \Gamma$, and an integer $k \geqq 2$ such that $H \sim k E+\Gamma,\left(E^{2}\right)=0,\left(\Gamma^{2}\right)=$ $-2,(E \cdot \Gamma)=1$. In this case, every member of $|H|$ is of the form $E^{\prime}+\Gamma$, where $E^{\prime}$ is a sum of $k$ effective divisors $E_{1}, \cdots, E_{k}$ such that $E_{i} \sim E$ for all $i$.

(2) Let $\left(H^{2}\right) \geqq 4$. Then $H$ is very ample if and only if

(i) there is no irreducible curve $E$ such that $\left(E^{2}\right)=0,(E \cdot H)=1,2$,

(ii) there is no irreducible curve $E$ such that $\left(E^{2}\right)=2, H \sim 2 E$, and

(iii) there is no irreducible curve $E$ such that $\left(E^{2}\right)=-2,(E \cdot H)=0$.

Proposition 6. Let $X, H, C$ be as in Remark 4. Then $H$ is very ample and $|C|$ contains an irreducible smooth member.

Proof. We will first check that $H$ satisfies the conditions (i)-(iii) in (2) of Theorem 5 . We denote by $\operatorname{disc}(A, B)$ the determinant of the intersection matrix of divisors $A$ and $B$. If there is a divisor $E$ such that $\left(E^{2}\right)=-2,(E \cdot H)=0$, then $\operatorname{disc}(E, H)=-8$ is divisible by $\operatorname{disc}(H, C)=$ $8(g-1)-d^{2}$. However, by $g \leqq d-3$, one has $d^{2} \geqq(g+3)^{2}>8 g$ and $\operatorname{disc}(H, C)<-8$. This is a contradiction. Thus (iii) is checked, (i) is checked in the same way, and (ii) is obvious because $H$ is a part of the basis of Pic $X$. Hence $H$ is very ample. Assume that $g \geqq 2$. Then we use (1) of Theorem 5 to show that $C$ is base point free. If $C$ is not base point free, then there is a divisor $E$ such that $\left(E^{2}\right)=0,(E \cdot C)=1$. Then $\operatorname{disc}(E, C)=-1$ is divisible by $\operatorname{disc}(H, C)$, which is a contradiction, as we have seen above. Thus $C$ is base point free and $|C|$ has an irreducible smooth member because $\left(C^{2}\right)>0$. Let $g=1$. Then the equation

$$
(x H+y C)^{2}=2 x(2 x+d y)=-2
$$

does not have integral solutions $x, y$, because $d \geqq g+3=4$.

Hence $X$ does not contain smooth rational curves by Remark 4, (4). By $\left(C^{2}\right)=0,|C|$ or $|-C|$ contains an effective member. By $(C \cdot H)=d>0$, $|C|$ contains an effective member $C_{0}$. Thus $C$ is numerically effective because otherwise $C_{0}$ contains an irreducible curve $Z \cong \boldsymbol{P}^{1}$, which is a contradiction. Hence by (1) of Theorem 5, $C$ is base point free, and $C$ is a multiple of an elliptic pencil. Since $C$ is a part of the basis of Pic $X,|C|$ is an elliptic pencil, and it contains a smooth elliptic curve. Let $g=0$. Since $\left(C^{2}\right)=-2$ and $(C \cdot H)>0$, one has $C \sim E+D$, where 
$E \cong P^{1}$ and $D$ is an effecitve divisor. Since $\operatorname{disc}(H, C)$ divides $\operatorname{disc}(H, E)$, one has $8+(C \cdot H)^{2} \leqq 8+(E \cdot H)^{2}$. Thus $(D \cdot H) \leqq 0$, and $D=0$. Hence $C=E$, and Proposition 6 is proved.

We can now finish the proof of the if-part $(\Leftarrow)$ of Theorem 1 . We use induction on $d$. We omit the proof for $(d, g)=(1,0),(2,0),(3,1)$, since they are well known. We may assume that $g<d^{2} / 8$, otherwise $C$ is given as a complete intersection. We may also assume that $g \geqq d-2$ by Remark 4 and Proposition 6. Thus as shown by the picture, one sees

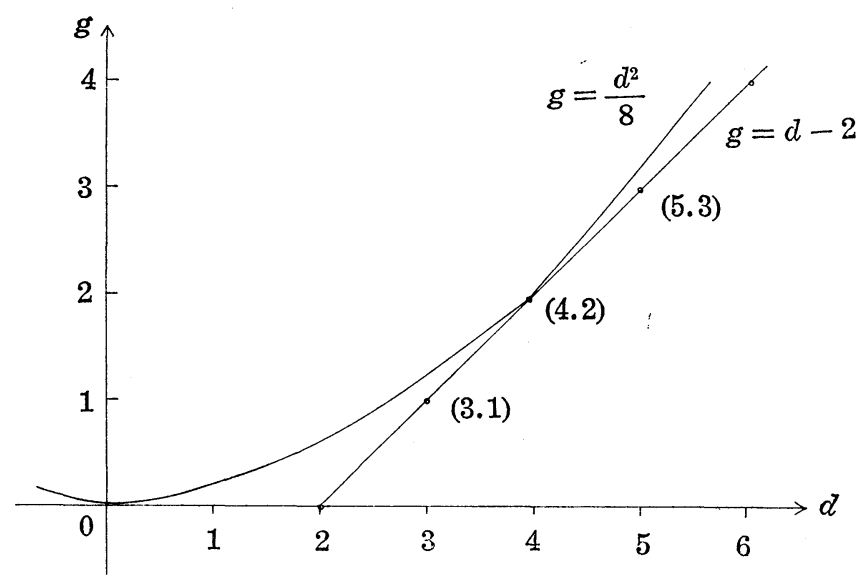

$d \geqq 6$. First, we assume that $(d, g) \neq(9,10)$. Let $d^{\prime}=d-4$, and $g^{\prime}=$ $g-d+2$. Then $d^{\prime 2}-8 g^{\prime}=d^{2}-8 g>0$ and $\left(d^{\prime}, g^{\prime}\right) \neq(5,3)$. Thus by the induction hypothesis, there exist a non-singular quartic $X^{\prime}$ and a non-singular curve $C^{\prime}$ on it of degree $d^{\prime}$ and genus $g^{\prime}$. Let $H^{\prime}$ be an irreducible hyperplanesection of $X^{\prime}$, and $C=C^{\prime}+H^{\prime}$. Since $d^{\prime}=d-4$ $\geqq 2$, one sees $\left(C \cdot C^{\prime}\right)=2\left(g^{\prime}-1\right)+d^{\prime} \geqq 0$, and $C$ is numerically effective. Since $\left(H^{\prime 2}\right)=4, C$ is base point free by (1) of Theorem 5. If we denote by the same $C$, a smooth member of $|C|$, then $C$ has degree $d$ and genus g. Thus $C$ and $X$ are the required pair for $d$, g. For $(d, g)=(9,10)$, let $d^{\prime}=1, g^{\prime}=0$, and $C^{\prime}$ a straight line on a smooth quartic surface $X^{\prime}$. Let $H^{\prime}$ be an irreducible hyperplanesection of $X$ and $C=C^{\prime}+2 H^{\prime}$. Then, one sees that $C, X$ are the required pair as in the above argument. This proves Theorem 1 .

\section{REFERENCES}

[GP] L. Gruson and C. Peskin, Genre des courbes algébrique de l'espace projectif (II), Ann. Sci. Ecole Norm. Sup., Paris, $4^{\circ}$ série, t. 15 (1982), 401-418.

[K] K. Kodaira, On the structure of compact complex analytic surfaces, I, Amer. J. 
Math., 86 (1964), 751-798.

[MM] S. Mori and S. Mukai, The uniruledness of the moduli space of curves of genus 11, to appear in the proceedings of Japan France symposium on algebraic geometry, 1982.

[SD] B. Saint-Donat, Projective models of $K-3$ surfaces, Amer. J. Math., 96 (1974), $602-639$.

[SI] T. Shioda and H. Inose, On singular $K 3$ surfaces, Complex analysis and algebraic geometry, Iwanami Shoten, Cambridge University Press, 1977, 119-136.

Department of Mathematics

Nagoya University

Chikusa-ku, Nagoya 464

Japan 